\title{
Comparison Between Robotic and Laparoscopic or Open Anastomoses: A Systematic Review and Meta-Analysis
}

This article was published in the following Dove Press journal: Robotic Surgery: Research and Reviews

\section{loannis D Kostakis (D* \\ Harkiran Sran* \\ Raphael Uwechue \\ Pankaj Chandak (D) \\ Jonathon Olsburgh \\ Nizam Mamode \\ loannis Loukopoulos \\ Nicos Kessaris (D)}

Department of Nephrology and Transplantation, Guy's Hospital, Guy's and St Thomas' NHS Foundation Trust, London, UK

*These authors contributed equally to this work
Correspondence: Nicos Kessaris Department of Nephrology and

Transplantation, Guy's Hospital, Guy's and St Thomas' NHS Foundation Trust, Great Maze Pond, London SEI 9RT, UK

Tel +447774335I54

Email Nicos.Kessaris@gstt.nhs.uk
Introduction: Robotic surgery has been increasingly used in fashioning various surgical anastomoses. Our aim was to collect and analyze outcomes related to anastomoses performed using a robotic approach and compare them with those done using laparoscopic or open approaches through meta-analysis.

Methods: A systematic review was conducted for articles comparing robotic with laparoscopic and/or open operations (colectomy, low anterior resection, gastrectomy, Roux-en-Y gastric bypass (RYGB), pancreaticoduodenectomy, radical cystectomy, pyeloplasty, radical prostatectomy, renal transplant) published up to June 2019 searching Medline, Scopus, Google Scholar, Clinical Trials and the Cochrane Central Register of Controlled Trials. Studies containing information about outcomes related to hand-sewn anastomoses were included for meta-analysis. Studies with stapled anastomoses or without relevant information about the anastomotic technique were excluded. We also excluded studies in which the anastomoses were performed extracorporeally in laparoscopic or robotic operations.

Results: We included 83 studies referring to the aforementioned operations (4 randomized controlled and 79 non-randomized, 10 prospective and 69 retrospective) apart from colectomy and low anterior resection. Anastomoses done using robotic instruments provided similar results to those done using laparoscopic or open approach in regards to anastomotic leak or stricture. However, there were lower rates of stenosis in robotic than in laparoscopic RYGB $(p=0.01)$ and in robotic than in open radical prostatectomy $(\mathrm{p}<0.00001)$. Moreover, all anastomoses needed more time to be performed using the robotic rather than the open approach in renal transplant $(p \leq 0.001)$. Conclusion: Robotic anastomoses provide equal outcomes with laparoscopic and open ones in most operations, with a few notable exceptions.

Keywords: anastomosis, hand-sewn, leak, stenosis, stricture, robotic, laparoscopic, open

\section{Introduction}

The introduction of laparoscopic techniques is considered to be one of the most prominent changes in surgical practice in the last decade of the twentieth century. ${ }^{1}$ Since the first laparoscopic cholecystectomy in 1987, minimally invasive approaches have become the mainstay of most abdominal surgical procedures. The benefits of minimally invasive surgery are well established and include reduced analgesic requirements, reduced wound-related complications, shorter length of hospital stay, and faster return to normal daily activities. ${ }^{2}$

Laparoscopic surgery, however, has its technical limitations. As described by Ruurda et al, open procedures offer the surgeon unlimited flexibility in his/her body, arm, and 
hand positions. A surgeon's actions can be controlled by visual and haptic feedback. In laparoscopic surgery, however, natural dexterity is compromised by the restricted degree of motion in laparoscopic instruments. ${ }^{1}$ Hand-eye coordination is reduced by the need to move an instrument in the opposite direction from the desired target on the monitor. Furthermore, depth perception is compromised by the two-dimensional image, and the need to rely on an assistant to operate the camera takes away the surgeon's control over the field of view. ${ }^{2}$ Most importantly, the length of rigid laparoscopic instruments results in the ready transmission and exaggeration of tiny movements from the surgeon, making delicate procedures, particularly fine anastomoses difficult. ${ }^{3}$ As a result, although the majority of abdominal general surgical operations can be performed laparoscopically, techniques for more complex surgery are less easily reproducible and left in the hands of only a limited number of experts. ${ }^{1}$ Open approaches for complex operations, where delicate and accurate anastomoses with minimal risk of complication are of critical importance, have hence remained the gold standard over the last 60 years. ${ }^{4}$

The introduction of the da Vinci ${ }^{\circledR}$ Surgical System (Intuitive Surgical Inc., Sunnyvale, CA, US) in 2000, however, has allowed some of the technical limitations of laparoscopic surgery to be overcome. One of the main advantages is that of enhanced dexterity (increased degrees of freedom of movement) in the instruments and filtering of tremor, which enables the surgeon to operate in a similar manner to open surgery, thus enabling fine microsurgery and microanastomoses to be performed. ${ }^{1}$ Other advantages include a three-dimensional view of the operative field, which allows for better depth perception, the ability of the surgeon to control of the view of the operative field, and an ergonomically designed workstation where the surgeon assumes a comfortable sitting position. ${ }^{2}$

Since its introduction almost 20 years ago, robotic surgery has been successfully applied to key colorectal, gastric, pancreatic, urological and transplantation procedures where anastomoses form a critical part of the operation. The aim of this study was to review the current literature surrounding robotic surgery in the abdomen, with particular reference to a comparison between robotically fashioned anastomoses and similar anastomoses performed via a laparoscopic and/or open approach.

\section{Methods}

\section{Search Strategy}

A systematic review and meta-analysis were conducted in accordance with the Preferred Reporting Items for
Systematic Reviews and Meta-Analysis (PRISMA) guidelines. ${ }^{5}$ We systematically searched the following databases for articles published up to June 2019: Medline, Scopus, Google Scholar, Clinical Trials and Cochrane Central Register of Controlled Trials. We used the following search terms: "robotic", "robotic-assisted", "robot-assisted", "colectomy", "low anterior resection", "anterior resection", "rectal resection", "gastrectomy", "gastric bypass", "pancreaticoduodenectomy", "Whipple", "cystectomy", "ileal conduit", "neobladder", "pyeloplasty", "prostatectomy", "renal transplant", "kidney transplant", combined with the Boolean operators AND, OR.

\section{Inclusion and Exclusion Criteria}

We included only original articles written in English that compared robotic with laparoscopic and/or open procedures regarding the aforementioned types of operations, included data about anastomotic leak, anastomotic stricture and/or anastomotic time and the anastomoses were hand-sewn. We excluded reviews, case reports, congress abstracts, animal studies, original articles referring only to robotic operations without comparing them with either laparoscopic or open ones and original articles comparing robotic with laparoscopic and/or open operations, but without having data about anastomotic leak, anastomotic stricture and/or anastomotic time or without the anastomoses being hand-sewn or with the anastomoses performed extracorporeally in laparoscopic or robotic cases, and articles written in languages other than English.

\section{Review and Analysis}

We extracted data about patients' number, gender and age, type of operation, approach (robotic, laparoscopic or open), anastomotic technique, anastomotic leak, anastomotic stricture and anastomotic time.

\section{Statistical Analysis}

Meta-analysis was performed using Review Manager Version 5.3. Dichotomous variables were assessed using risk ratio (RR), whereas continuous variables were assessed using mean difference. The random-effects model was chosen due to the heterogeneity among the included studies. Comparisons between dichotomous or continuous variables were made with the inverse variance method. Statistical heterogeneity was assessed with the Higgin's $\mathrm{I}^{2}$ statistic. Ninety-five percent confidence intervals (CI) were noted for all results. Results were considered statistically significant if p-value was less than 0.05 . 


\section{Results}

\section{Search Results}

The initial database search yielded 510 studies comparing robotic with laparoscopic and/or open operations in regards to the aforementioned types of procedures. Out of the initial 510 articles, 261 were excluded due to data unavailability about anastomotic leak, anastomotic stricture and/or anastomotic time. Out of the remaining 249 articles, 158 were excluded due to anastomotic techniques involving stapling devices or extracorporeally performed anastomoses in laparoscopic or robotic cases. Finally, another 8 studies were excluded due to overlapping cases, leaving 83 studies to be included in our analysis ( 4 randomized controlled and 79 non-randomized, 10 prospective and 69 retrospective). In particular, the distribution of included articles according to the exact type of operation was the following: colectomy: 0 , low anterior resection: 0, gastrectomy: 4, Roux-en-Y gastric bypass (RYGB): 5 , pancreaticoduodenectomy: 16, radical cystectomy: 1 , pyeloplasty: 20 , radical prostatectomy: 36 , renal transplant: 1. Figure 1 shows the study flowchart.

\section{Gastrectomy}

Four studies referring to gastrectomy were taken into account for our analysis, including 689 adult patients in

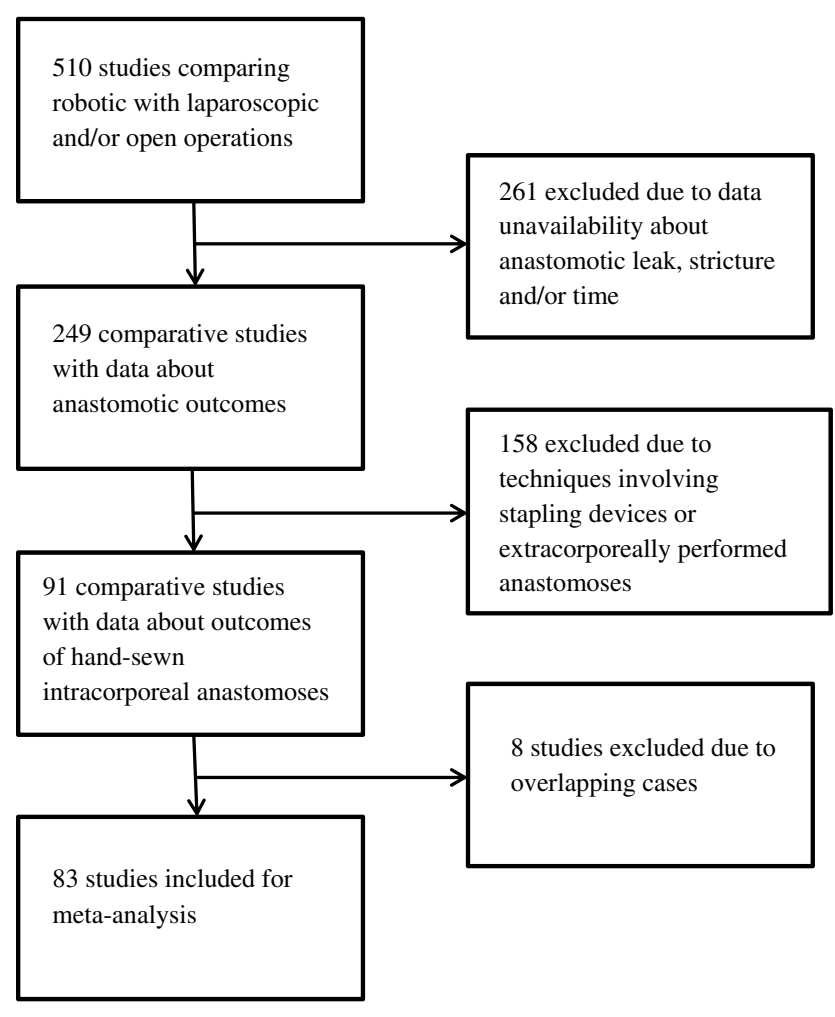

Figure I Study flowchart. total (451 men, 238 women). All studies included both total and subtotal gastrectomies. ${ }^{6-9}$ There was one prospective randomized study conducted in China comparing robotic with laparoscopic gastrectomy. There were only two cases of anastomotic leak in the laparoscopic group (61 patients), while there were no cases of anastomotic leak in the robotic group (102 patients) $(\mathrm{p}=0.139)^{7}$

The other three articles compared robotic with open gastrectomy and these included two retrospective nonrandomized studies, one from Spain $^{6}$ and one from Italy, ${ }^{8}$ and one prospective randomized study from China. ${ }^{9}$ There was no significant difference in the rates of anastomotic leak between the two treatment groups [robotic: 5/231 (2.2\%), open: $13 / 295$ (4.4\%), RR: $0.6,95 \%$ CI: 0.19 to 1.94, $\left.\mathrm{p}=0.4 ; \mathrm{I}^{2}: 7 \%, \mathrm{p}=0.34\right]^{6,8,9}$ (Figure 2). Data about anastomotic stricture were available only in one study, in which there was only one case of anastomotic stenosis in the robotic group (20 patients), whereas there was no similar case in the open group (19 patients) $(p=1){ }^{6}$

\section{Roux-En-Y Gastric Bypass}

Five studies referring to RYGB were considered for our analysis, which included 2155 adult patients in total (490 men, 1665 women). ${ }^{10-14}$ Out of these five studies, one was a prospective randomized trial conducted in the USA comparing robotic with laparoscopic RYGB, ${ }^{14}$ three were retrospective non-randomized done in the USA comparing again robotic with laparoscopic RYGB, ${ }^{10,11,13}$ and one was a retrospective non-randomized Swiss study with three treatment arms (robotic, laparoscopic, open). ${ }^{12}$

There was no statistically significant difference between robotic and laparoscopic RYGB in regards to anastomotic leak [robotic: $1 / 527(0.2 \%)$, laparoscopic: 14/588 (2.4\%), RR: $0.22,95 \%$ CI: 0.04 to $\left.1.38, \mathrm{p}=0.11 ; \mathrm{I}^{2}: 7 \%, \mathrm{p}=0.34\right]^{10-14}$ (Figure 3). However, there was an advantage of the robotic approach over the laparoscopic one in terms of anastomotic stricture [robotic: $0 / 381(0 \%)$, laparoscopic: $24 / 542(4.4 \%)$, RR: $0.07,95 \%$ CI: 0.01 to $0.54, p=0.01 ; I^{2}: 0 \%$, $\mathrm{p}=0.72]^{10,12-14}$ (Figure 4). The only study that compared robotic with open procedures concluded that there is no statistically significant difference between robotic and open operations as far as anastomotic leak [robotic: 0/143 (0\%), open: $10 / 524(1.9 \%), \mathrm{p}=0.21$ ] or stricture [robotic: $0 / 143$ (0\%), open: 6/524 (1.1\%), $\mathrm{p}=0.23$ ] are concerned. ${ }^{12}$

\section{Pancreaticoduodenectomy}

Sixteen studies referring to pancreaticoduodenectomy were taken into account for our analysis, including 12,529 adult 


\begin{tabular}{|c|c|c|c|c|c|c|c|c|c|c|}
\hline Study or Subgroup & \multicolumn{2}{|c|}{ Robotic } & \multicolumn{2}{|c|}{ Open } & Weight & $\begin{array}{c}\text { Risk Ratio } \\
\text { IV, Random, } 95 \% \mathrm{Cl} \\
\end{array}$ & \multicolumn{4}{|c|}{$\begin{array}{c}\text { Risk Ratio } \\
\text { IV, Random, } 95 \% \mathrm{Cl} \\
\end{array}$} \\
\hline Caruso 2019 & 0 & 20 & 2 & 19 & $14.9 \%$ & $0.19[0.01,3.73]$ & & $\rightarrow$ & & \\
\hline Solaini 2019 & 1 & 60 & 8 & 131 & $30.2 \%$ & $0.27[0.03,2.13]$ & & & - & \\
\hline Wang 2016 & 4 & 151 & 3 & 145 & $54.9 \%$ & $1.28[0.29,5.62]$ & & & & \\
\hline Total $(95 \% \mathrm{Cl})$ & & 231 & & 295 & $100.0 \%$ & $0.60[0.19,1.94]$ & & & & \\
\hline Total events & 5 & & 13 & & & & & & & \\
\hline $\begin{array}{l}\text { Heterogeneity: Tau } \\
\text { Test for overall effec }\end{array}$ & $\begin{array}{l}0.08 ; \mathrm{ch} \\
z=0.84\end{array}$ & $\begin{array}{l}P=2.1 \\
P=0.4\end{array}$ & $\begin{array}{l}4, \mathrm{df}=2(\mathrm{C} \\
0)\end{array}$ & $=0.3$ & $4) ;\left.\right|^{2}=7 \%$ & & 0.005 & $\begin{array}{c}0.1 \\
\text { Favours robotic }\end{array}$ & Favours open & 200 \\
\hline
\end{tabular}

Figure 2 Comparison between robotic and open gastrectomy (anastomotic leak).

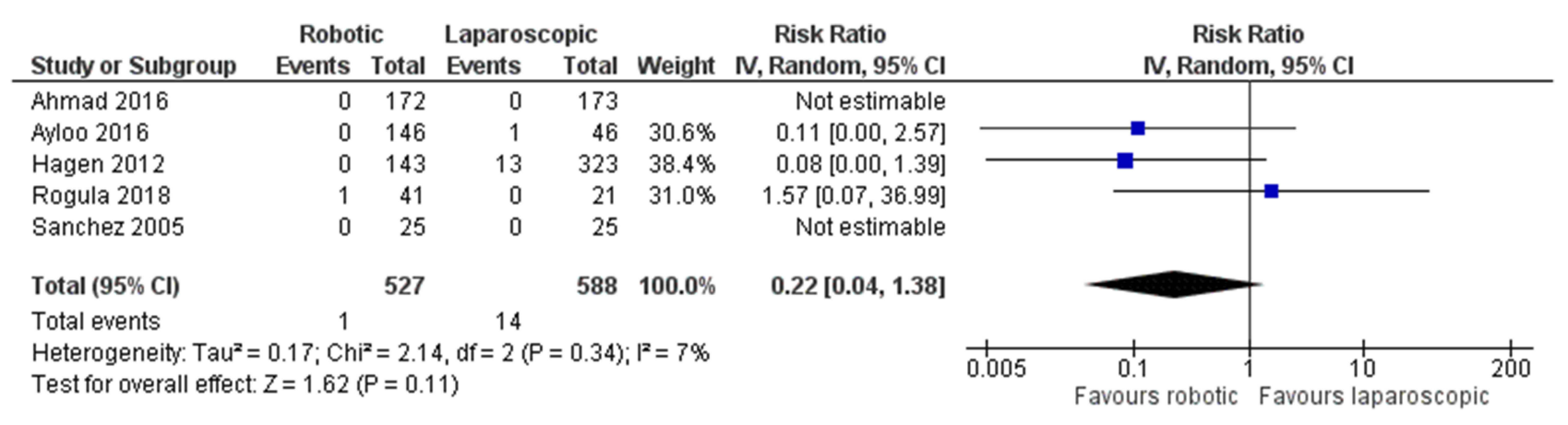

Figure 3 Comparison between robotic and laparoscopic RYGB: anastomotic leak.

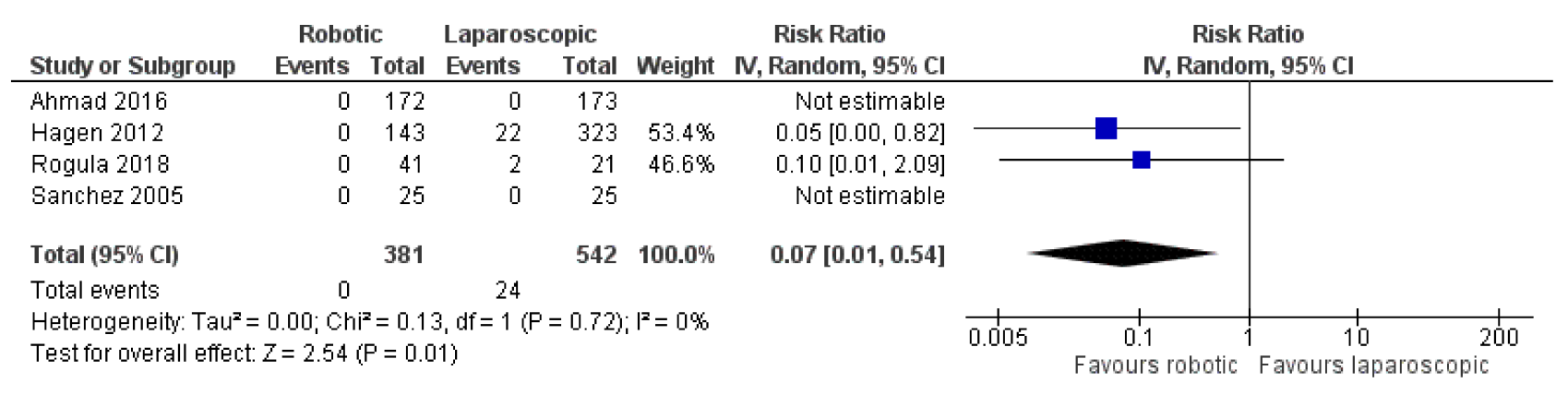

Figure 4 Comparison between robotic and laparoscopic RYGB: anastomotic stricture.

patients in total (6627 men, 5902 women). ${ }^{15-30}$ All included studies were retrospective non-randomized, ${ }^{15-19,21-30}$ apart from one prospective non-randomized study from China. ${ }^{20}$ Nine articles were from the USA, ${ }^{15,16,18,19,24-26,29,30}$ five from China, ${ }^{20,22,23,27,28}$ one from Italy $^{17}$ and one from South Korea. ${ }^{21}$ Three articles compared robotic with laparoscopic operations, ${ }^{23,25,27} 12$ articles compared robotic with open procedures, ${ }^{15-22,24,26,28,30}$ and one article compared all three options. $^{29}$

No significant difference was noted between robotic and laparoscopic pancreaticoduodenectomy in regards to pancreatic leak [robotic: 93/451 (20.6\%), laparoscopic: 107/ 560 (19.1\%), RR: $1.08,95 \%$ CI: 0.85 to $1.39, \mathrm{p}=0.52 ; \mathrm{I}^{2}$ : $0 \%, \mathrm{p}=0.72]^{23,25,27,29}$ (Figure 5) or bile leak [robotic: $4 / 47$
(8.5\%), laparoscopic: 7/45 (15.6\%), RR: 0.54, 95\% CI: 0.17 to $\left.1.71, \mathrm{p}=0.3 ; \mathrm{I}^{2}: 0 \%, \mathrm{p}=0.94\right]^{23,27}$ (Figure 6). Similarly, no significant difference was detected between robotic and open pancreaticoduodenectomy in regards to pancreatic leak [robotic: 176/1086 (16.2\%), open: 1679/10,526 (16\%), RR: $0.93,95 \% \mathrm{CI}: 0.7$ to $1.24, \mathrm{p}=0.64$ ] [although there was significant heterogeneity among studies $\left(\mathrm{I}^{2}\right.$ : $54 \%$, $\mathrm{p}=0.01)]^{15-22,24,26,28-30}$ (Figure 7), bile leak [robotic: 10/ 227 (4.4\%), open: 18/491 (3.7\%), RR: 1.27, 95\% CI: 0.58 to $\left.2.78, \mathrm{p}=0.55 ; \mathrm{I}^{2}: 7 \%, \mathrm{p}=0.37\right]^{15,18-22}$ (Figure 8 ) or leak from gastrointestinal anastomoses [robotic: 2/82 (2.4\%), open: 5/169 (3\%), RR: 1, 95\% CI: 0.22 to $4.57, \mathrm{p}=1$; $\mathrm{I}^{2}$ : $0 \%, 0.53]^{15,20}$ (Figure 9). There were no available data regarding anastomotic strictures. 


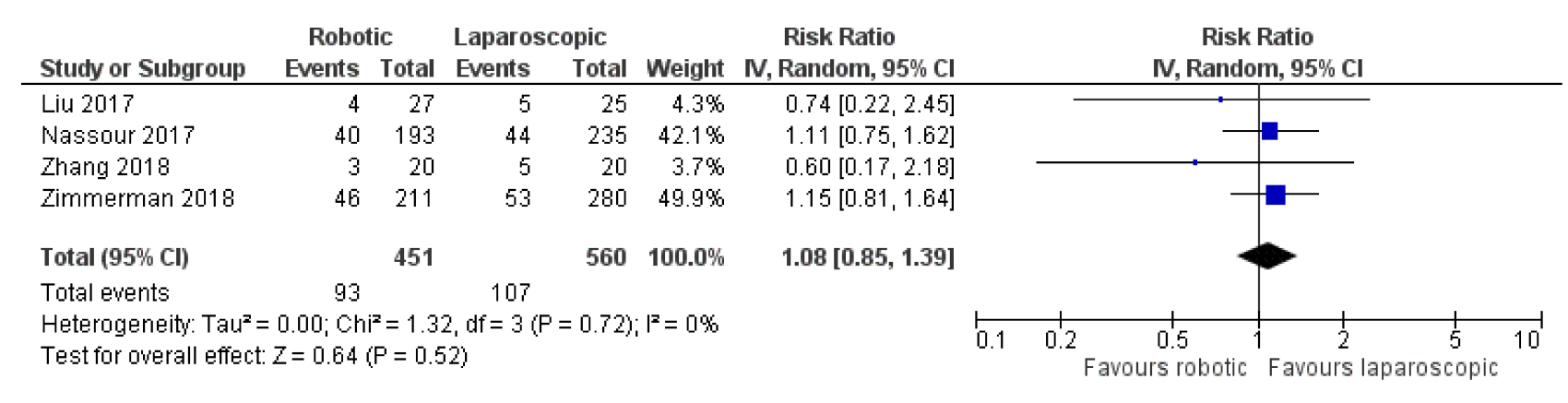

Figure 5 Comparison between robotic and laparoscopic pancreaticoduodenectomy: pancreatic leak.

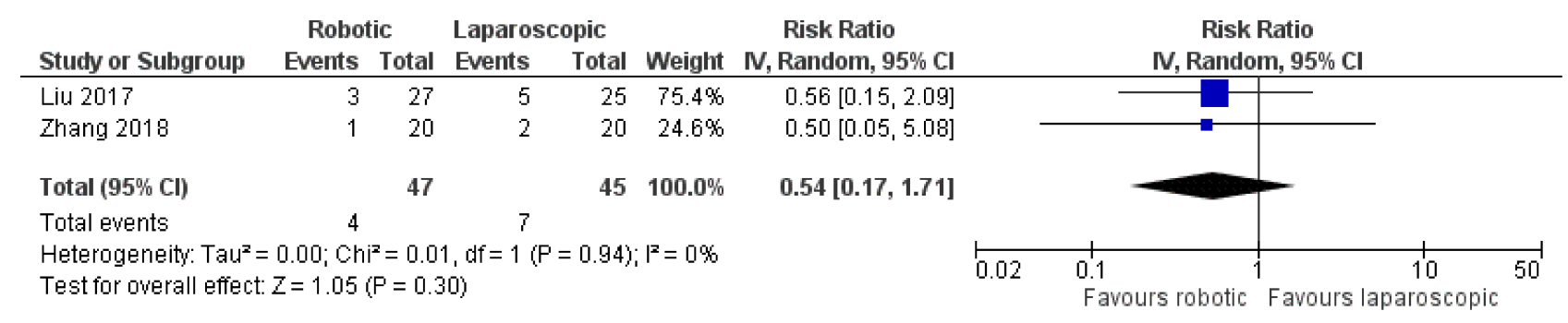

Figure 6 Comparison between robotic and laparoscopic pancreaticoduodenectomy: bile leak.

\begin{tabular}{|c|c|c|c|c|c|c|c|c|c|c|}
\hline \multirow[b]{2}{*}{ Study or Subgroup } & \multicolumn{2}{|c|}{ Robotic } & \multicolumn{2}{|c|}{ Open } & \multirow[b]{2}{*}{ Weight } & \multicolumn{2}{|l|}{ Risk Ratio } & \multirow{2}{*}{\multicolumn{2}{|c|}{$\begin{array}{c}\text { Risk Ratio } \\
\text { IV, Random, 95\% Cl }\end{array}$}} & \\
\hline & Events & Total & Events & Total & & IV, Random, 95\% Cl & & & & \\
\hline Baker 2016 & 1 & 22 & 6 & 49 & $1.7 \%$ & $0.37[0.05,2.90]$ & & & & \\
\hline Bao 2014 & 8 & 28 & 10 & 28 & $7.6 \%$ & $0.80[0.37,1.72]$ & & & & \\
\hline Boggi 2016 & 28 & 83 & 6 & 36 & $7.3 \%$ & $2.02[0.92,4.46]$ & & & & \\
\hline Buchs 2011 & 8 & 44 & 8 & 39 & $6.4 \%$ & $0.89[0.37,2.14]$ & & & & \\
\hline Chalikonda 2012 & 4 & 30 & 5 & 30 & $4.1 \%$ & $0.80[0.24,2.69]$ & & & & \\
\hline Chen 2015 & 8 & 60 & 29 & 120 & $8.2 \%$ & $0.55[0.27,1.13]$ & & & & \\
\hline Kim 2018 & 3 & 51 & 19 & 186 & $4.3 \%$ & $0.58[0.18,1.87]$ & & & & \\
\hline Lai 2012 & 7 & 20 & 12 & 67 & $7.4 \%$ & $1.95[0.89,4.29]$ & & & & \\
\hline Wc: willan 2017 & 16 & 185 & 351 & 2661 & $11.6 \%$ & $0.66[0.41,1.06]$ & & & & \\
\hline Varley 2019 & 16 & 133 & 35 & 149 & $10.5 \%$ & $0.51[0.30,0.88]$ & & & & \\
\hline Zhou 2011 & 2 & 8 & 3 & 8 & $2.9 \%$ & $0.67[0.15,2.98]$ & & & & \\
\hline Zimmerman 2018 & 46 & 211 & 1121 & 6336 & $15.1 \%$ & $1.23[0.95,1.60]$ & & & & \\
\hline Zureikat 2018 & 29 & 211 & 74 & 817 & $12.8 \%$ & $1.52[1.02,2.27]$ & & & & \\
\hline Total $(95 \% \mathrm{CI})$ & & 1086 & & 10526 & $100.0 \%$ & $0.93[0.70,1.24]$ & & & & \\
\hline Total events & 176 & & 1679 & & & & & & & \\
\hline $\begin{array}{l}\text { Heterogeneity: Tau } \\
\text { Test for overall effec }\end{array}$ & $\begin{array}{l}0.12 ; \text { Chi } \\
z=0.476\end{array}$ & $\begin{array}{l}P^{2}=26 \\
P=0.6\end{array}$ & $\begin{array}{l}31, d f=1 \\
44)\end{array}$ & $P=0$ & $010) ;\left.\right|^{2}=$ & $54 \%$ & 0.0 & $\begin{array}{l}0.1 \\
\text { Favours robotic }\end{array}$ & Favours open & 50 \\
\hline
\end{tabular}

Figure 7 Comparison between robotic and open pancreaticoduodenectomy: pancreatic leak.

\section{Radical Cystectomy}

Only one retrospective non-randomized study concerning radical cystectomy was included in our analysis, which was conducted in South Korea and compared robotic with open radical cystectomy. ${ }^{31}$ It included 139 adult patients (116 men, 23 women). Out of the 139 cases, a neobladder with handsewn urethroneovesical anastomosis was formed in 41 . There were 19 cases of open and 22 cases of robotic radical cystectomy. No postoperative anastomotic leak in the open group [0/
$19(0 \%)$, but there were three cases of postoperative anastomotic leak in the robotic group [3/22 (13.6\%)]. However, this difference was not significant when we compared the two groups with Fisher's exact test $(\mathrm{p}=0.235)$. There was no available information about anastomotic strictures. ${ }^{31}$

\section{Pyeloplasty}

Twenty studies referring to pyeloplasty were considered for our analysis, which included 1158 patients in total. ${ }^{32-51}$ Ten 


\begin{tabular}{|c|c|c|c|c|c|c|c|c|c|c|}
\hline \multirow[b]{2}{*}{ Study or Subgroup } & \multicolumn{2}{|c|}{ Robotic } & \multicolumn{2}{|c|}{ Open } & \multirow[b]{2}{*}{ Weight } & \multirow{2}{*}{$\begin{array}{c}\text { Risk Ratio } \\
\text { IV, Random, } 95 \% \mathrm{Cl}\end{array}$} & \multirow{2}{*}{\multicolumn{4}{|c|}{$\begin{array}{c}\text { Risk Ratio } \\
\text { IV, Random, } 95 \% \mathrm{Cl} \\
\end{array}$}} \\
\hline & Events & Total & Events & Total & & & & & & \\
\hline Baker 2016 & 0 & 22 & 2 & 49 & $6.6 \%$ & $0.43[0.02,8.70]$ & & & & \\
\hline Buchs 2011 & 1 & 44 & 2 & 39 & $10.5 \%$ & $0.44[0.04,4.70]$ & & & & \\
\hline Chalikonda 2012 & 0 & 30 & 2 & 30 & $6.6 \%$ & $0.20[0.01,4.00]$ & & & & \\
\hline Chen 2015 & 5 & 60 & 8 & 120 & $43.2 \%$ & $1.25[0.43,3.66]$ & & & & \\
\hline Kim 2018 & 1 & 51 & 0 & 186 & $5.9 \%$ & $10.79[0.45,260.94]$ & & & & \\
\hline Lai 2012 & 3 & 20 & 4 & 67 & $27.2 \%$ & $2.51[0.61,10.30]$ & & & - & \\
\hline Total $(95 \% \mathrm{Cl})$ & & 227 & & 491 & $100.0 \%$ & $1.27[0.58,2.78]$ & & & & \\
\hline Total events & 10 & & 18 & & & & & & & \\
\hline $\begin{array}{l}\text { Heterogeneity: Tauz } \\
\text { Test for overall effec }\end{array}$ & $\begin{array}{l}0.07 ; \text { Chi } \\
z=0.60\end{array}$ & $\begin{array}{l}z=5.3 \\
P=0.5\end{array}$ & $5, \mathrm{df}=5($ & $P=0.3$ & $7) ; 1^{2}=7 \%$ & & 0.005 & $\begin{array}{c}0.1 \\
\text { Favours robotic }\end{array}$ & $\begin{array}{c}10 \\
\text { Favours open }\end{array}$ & 200 \\
\hline
\end{tabular}

Figure 8 Comparison between robotic and open pancreaticoduodenectomy: bile leak.

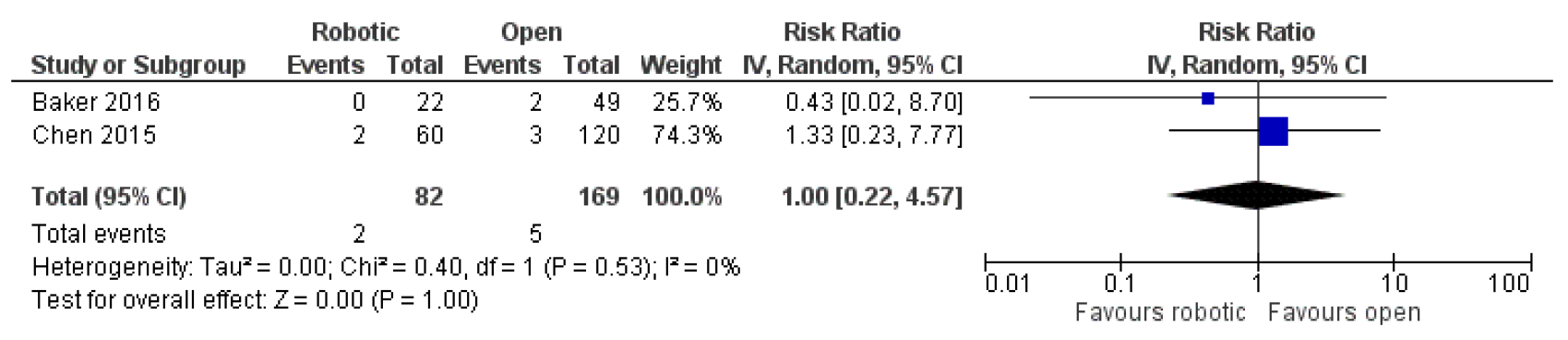

Figure 9 Comparison between robotic and open pancreaticoduodenectomy: leak from gastrointestinal anastomoses.

articles referred to paediatric patients, ${ }^{32,33,38,43,45-49,51}$ five articles referred to adult patients ${ }^{34,35,37,42,44}$ and five articles referred to both paediatric and adult patients. ${ }^{36,39-41,50}$ Gender distribution was mentioned in 17 articles, ${ }^{32-34,36,37,40-51}$ which included 625 male and 432 female patients in total. All included studies were retrospective nonrandomized, ${ }^{32-41,43-51}$ with the exception of one, which was prospective non-randomized. ${ }^{42}$ Eleven studies were from the USA, ${ }^{32,33,35,36,38,42,44,45,47,50,51}$ one from Italy, ${ }^{37}$ one from Switzerland, ${ }^{48}$ one from Israel, ${ }^{43}$ one from Turkey, ${ }^{34}$ two from India, ${ }^{39,41}$ two from China ${ }^{40,49}$ and one from South Korea. ${ }^{46}$ Fourteen articles compared robotic with laparoscopic operations, ${ }^{35-45,48-50}$ four articles compared robotic with open procedures, ${ }^{32,33,47,51}$ and two articles compared all three options. $^{34,46}$

There was no significant difference between robotic and laparoscopic pyeloplasty concerning anastomotic leak [robotic: 13/388 (3.4\%), laparoscopic: 9/291 (3.1\%), RR: $1.0395 \%$ CI: 0.46 to $2.32, p=0.95 ; \mathrm{I}^{2}: 0 \%$, $\mathrm{p}=0.93]^{34-38,40-46,48-50}$ (Figure 10) or anastomotic stricture/failure [robotic: 4/403 (1\%), laparoscopic: 10/309 (3.2\%), RR: $0.53,95 \%$ CI: 0.2 to $1.44, \mathrm{p}=0.21 ; \mathrm{I}^{2}: 0 \%$, $\mathrm{p}=0.99]^{34-37,39-46,48-50}$ (Figure 11). Similarly, no significant difference was detected between robotic and open pyeloplasty regarding anastomotic leak [robotic: 1/100
(1\%), open: $7 / 278$ (2.5\%), RR: $0.94,95 \% \mathrm{CI}: 0.18$ to 4.85, $\left.\mathrm{p}=0.94 ; \mathrm{I}^{2}: 10 \%, \mathrm{p}=0.33\right]^{32-34,46,51}$ (Figure 12) or anastomotic stricture/failure [robotic: 3/133 (2.2\%), open: 8/311 (2.6\%), RR: $1,95 \%$ CI: 0.32 to $3.09, p=0.99 ; \mathrm{I}^{2}: 0 \%$, $\mathrm{p}=0.97]^{32-34,46,47,51}$ (Figure 13).

\section{Radical Prostatectomy}

Thirty-six studies referring to radical prostatectomy were taken into account for our analysis, including 40,313 adult male patients in total. ${ }^{52-87}$ Twenty-seven studies were retrospective non-randomized, ${ }^{52-57,60,62,63,65-70,72,73,75,76,78,79,81-84,86,87}$ eight studies were prospective non-randomized ${ }^{58,59,61,64,71,74,77,85}$ and one study was prospective randomized. ${ }^{80}$ Twelve studies were from the USA, $52,56,58,64,66,67,69,70,74,75,79,87$ one from the $\mathrm{UK}^{78}$ one from Germany, ${ }^{85}$ three from France, ${ }^{61,80,82}$ one from Belgium, ${ }^{53}$ three from Italy, ${ }^{62,71,81}$ one from Norway, ${ }^{68}$ two from Sweden, ${ }^{57,86}$ one from Switzerland, ${ }^{59}$ three from Australia, ${ }^{60,77,88}$ one from Canada, ${ }^{73}$ three from South Korea, ${ }^{65,83,84}$ one from Taiwan, ${ }^{76}$ one from Thailand ${ }^{54}$ and one from Venezuela, ${ }^{72}$ while one study was multinational. ${ }^{63}$ Thirteen articles compared robotic with laparoscopic operations, ${ }^{53,54,63,65,67,68,71,76,79-81,84,87} 19$ articles compared robotic with open procedures, $52,55-59,61,62,66,69,72-75,77,82,85,86$ and four articles compared all three options. ${ }^{60,70,78,83}$ 


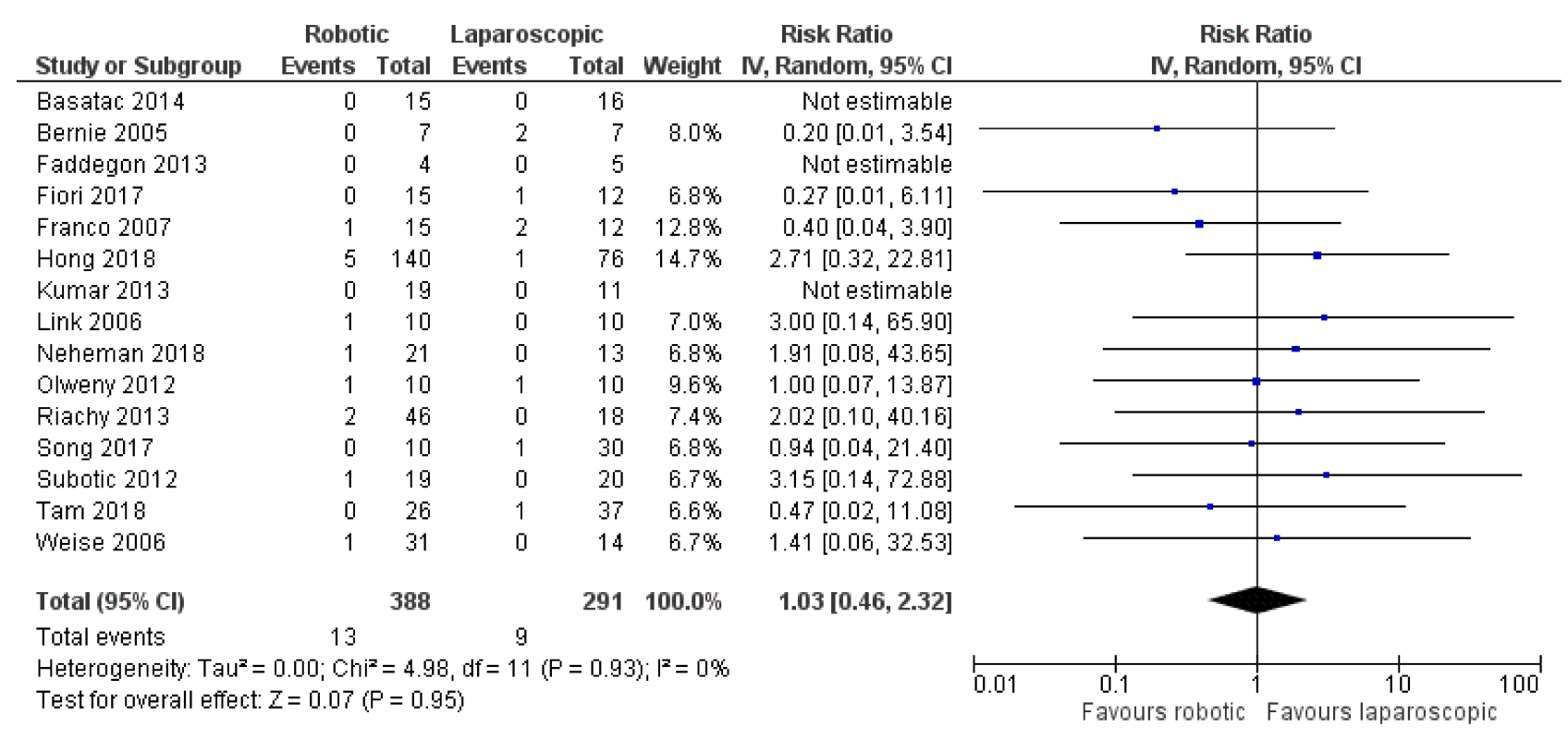

Figure 10 Comparison between robotic and laparoscopic pyeloplasty: anastomotic leak.

Robotic Laparoscopic Risk Ratio Study or Subgroup Events Total Events Total Weight IV, Random, 95\% Cl

\begin{tabular}{|c|c|c|c|c|c|}
\hline Basatac 2014 & 1 & 15 & 1 & 16 & $13.9 \%$ \\
\hline Bernie 2005 & 0 & 7 & 0 & 7 & \\
\hline Faddegon 2013 & 0 & 4 & 0 & 5 & \\
\hline Fiori 2017 & 0 & 15 & 0 & 12 & \\
\hline Hemal 2010 & 0 & 30 & 1 & 30 & $10.0 \%$ \\
\hline Hong 2018 & 0 & 140 & 1 & 76 & $9.8 \%$ \\
\hline Kumar 2013 & 0 & 19 & 0 & 11 & \\
\hline Link 2006 & 0 & 10 & 0 & 10 & \\
\hline Neheman 2018 & 1 & 21 & 1 & 13 & $13.9 \%$ \\
\hline olweny 2012 & 0 & 10 & 1 & 10 & $10.5 \%$ \\
\hline Riachy 2013 & 0 & 46 & 0 & 18 & \\
\hline Song 2017 & 0 & 10 & 2 & 30 & $11.4 \%$ \\
\hline Subotic 2012 & 0 & 19 & 0 & 20 & \\
\hline Tam 2018 & 1 & 26 & 3 & 37 & $20.5 \%$ \\
\hline Weise 2006 & 1 & 31 & 0 & 14 & $10.1 \%$ \\
\hline Total $(95 \% \mathrm{Cl})$ & \multicolumn{3}{|c|}{403} & 309 & $100.0 \%$ \\
\hline Total events & \multicolumn{5}{|c|}{10} \\
\hline $\begin{array}{l}\text { Heterogeneity: Ta } \\
\text { Test for overall ef }\end{array}$ & ch & & $=70$ & & $0 \%$ \\
\hline
\end{tabular}

$1.07[0.07,15.57]$

Not estimable

Not estimable

Not estimable

$0.33[0.01,7.87]$

$0.18[0.01,4.41]$

Not estimable

Not estimable

$0.62[0.04,9.07]$

$0.33[0.02,7.32]$

Not estimable

$0.56[0.03,10.85]$

Not estimable

$0.47[0.05,4.31]$

$1.41[0.06,32.53]$

\section{$0.53[0.20,1.44]$}

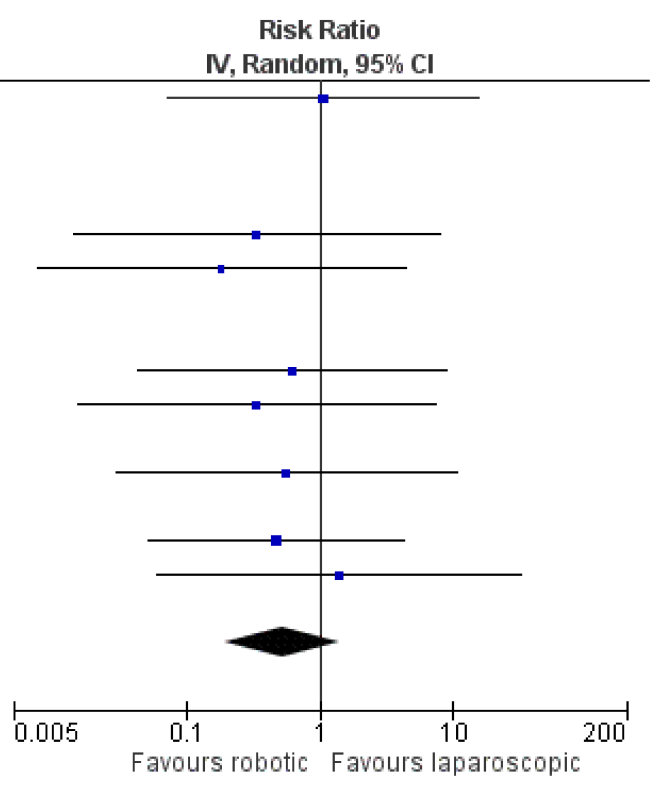

Figure I I Comparison between robotic and laparoscopic pyeloplasty: anastomotic stricture/failure.

Robotic

Open

Risk Ratio

Study or Subgroup Events Total Events Total Weight IV, Random, 95\% C

\begin{tabular}{lrrrrrr}
\hline Bansal 2014 & 1 & 9 & 2 & 61 & $43.9 \%$ & $3.39[0.34,33.67]$ \\
Barbosa 2013 & 0 & 58 & 2 & 154 & $27.0 \%$ & $0.53[0.03,10.78]$ \\
Basatac 2014 & 0 & 15 & 3 & 25 & $29.1 \%$ & $0.23[0.01,4.21]$ \\
Song 2017 & 0 & 10 & 0 & 30 & & Not estimable \\
Yee 2006 & 0 & 8 & 0 & 8 & & Not estimable \\
Total (95\% Cl) & \multicolumn{7}{c}{$\mathbf{1 0 0}$} & & 278 & $\mathbf{1 0 0 . 0} \%$ & $\mathbf{0 . 9 4}[0.18,4.85]$ \\
Total events & 1 & 7 & \\
Heterogeneity: Tau ${ }^{2}=0.23 ; \mathrm{Chi}^{2}=2.23, \mathrm{df}=2(\mathrm{P}=0.33) ; \mathrm{I}^{2}=10 \%$ & \\
Test for overall effect: $Z=0.08(\mathrm{P}=0.94)$ &
\end{tabular}

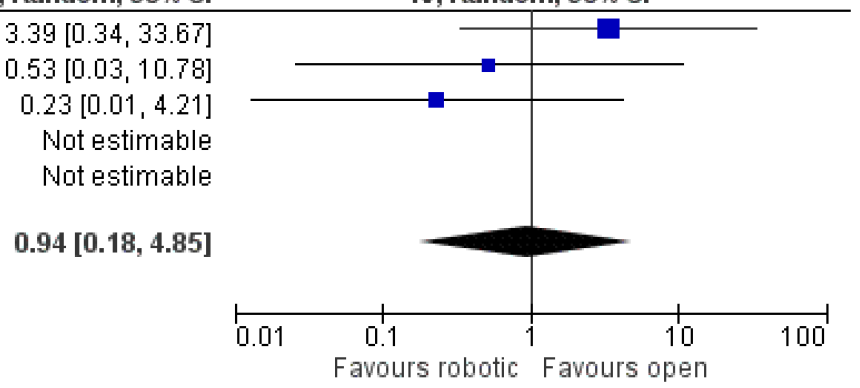

Figure 12 Comparison between robotic and open pyeloplasty: anastomotic leak. 


\begin{tabular}{|c|c|c|c|c|c|c|c|c|c|c|}
\hline \multirow[b]{2}{*}{ Study or Subgroup } & \multicolumn{2}{|c|}{ Robotic } & \multicolumn{2}{|c|}{ Open } & \multirow[b]{2}{*}{ Weight } & \multirow{2}{*}{$\begin{array}{c}\text { Risk Ratio } \\
\text { IV, Random, } 95 \% \mathrm{CI}\end{array}$} & \multirow{2}{*}{\multicolumn{4}{|c|}{$\begin{array}{c}\text { Risk Ratio } \\
\text { IV, Random, } 95 \% \mathrm{Cl} \\
\end{array}$}} \\
\hline & Events & Total & Events & Total & & & & & & \\
\hline Bansal 2014 & 0 & 9 & 1 & 61 & $13.1 \%$ & $2.07[0.09,47.28]$ & & & & \\
\hline Barbosa 2013 & 1 & 58 & 3 & 154 & $25.4 \%$ & $0.89[0.09,8.34]$ & & & & \\
\hline Basatac 2014 & 1 & 15 & 1 & 25 & $17.6 \%$ & $1.67[0.11,24.72]$ & & & & \\
\hline Song 2017 & 0 & 10 & 1 & 30 & $13.1 \%$ & $0.94[0.04,21.40]$ & & & & \\
\hline Sorensen 2011 & 1 & 33 & 1 & 33 & $17.2 \%$ & $1.00[0.07,15.33]$ & & & & \\
\hline Yee 2006 & 0 & 8 & 1 & 8 & $13.6 \%$ & $0.33[0.02,7.14]$ & & & & \\
\hline Total $(95 \% \mathrm{Cl})$ & & 133 & & 311 & $100.0 \%$ & $1.00[0.32,3.09]$ & & & & \\
\hline Total events & 3 & & 8 & & & & & & & \\
\hline $\begin{array}{l}\text { Heterogeneity: Tau } \\
\text { Test for overall effec }\end{array}$ & $\begin{array}{l}0.00 ; \text { Chi } \\
Z=0.01\end{array}$ & $\begin{array}{l}z=0.8 \\
P=0.9\end{array}$ & $\begin{array}{l}5, \mathrm{df}=50 \\
19)\end{array}$ & $=0.9$ & $7 i^{2}=0 \%$ & & 0.01 & $\begin{array}{c}0.1 \\
\text { Favours robotic }\end{array}$ & $\begin{array}{cc}10 \\
\text { Favours open }\end{array}$ & 100 \\
\hline
\end{tabular}

Figure 13 Comparison between robotic and open pyeloplasty: anastomotic stricture/failure.

There was no significant difference between robotic and laparoscopic radical prostatectomy in terms of leak from the vesicourethral anastomosis [robotic: 152/4601 (3.3\%), laparoscopic: $132 / 3913$ (3.4\%), RR: $0.92,95 \%$ CI: 0.56 to $1.51, \mathrm{p}=0.73$ ] [although there was significant heterogeneity among studies $\left.\left(\mathrm{I}^{2}: 57 \%, \mathrm{p}=0.008\right)\right]^{53,60,65,67,68,70,76,78-81,84,87}$ (Figure 14) or anastomotic stricture/contracture [robotic: 604907 (1.2\%), laparoscopic: 72/3669 (2\%), RR: $0.67,95 \%$ CI: 0.42 to $1.07, \quad \mathrm{p}=0.09 ; \mathrm{I}^{2}: 16 \%$, $\mathrm{p}=0.29]^{54,63,65,67,68,70,71,76,79,80,83,87}$ (Figure 15). When we compared robotic with open radical prostatectomy, no significant difference was found in regards to leak from the vesicourethral anastomosis [robotic: 119/6097 (2\%), open: 185/8323 (2.2\%), RR: $0.76,95 \%$ CI: 0.44 to 1.34 , $\mathrm{p}=0.35]^{52,56-61,64,70,72-75,77,78,82,86}$ (Figure 16), but there were lower rates of anastomotic stricture/contracture in robotic approach [robotic: 180/9626 (1.9\%), open: 1125/
12,102 (9.3\%), RR: $0.44, \quad 95 \%$ CI: 0.32 to 0.62 , $\mathrm{p}<0.00001]^{55,56,58,61,62,66,69,70,72,73,75,77,83,85,86}$ (Figure 17). However, we have to mention that there was significant heterogeneity among the included studies for both comparisons between robotic and open procedures (anastomotic leak: $\mathrm{I}^{2}: 73 \%, \mathrm{p}<0.00001$, anastomotic stricture/contracture: $\left.\mathrm{I}^{2}: 45 \%, \mathrm{p}=0.03\right)$.

\section{Renal Transplant}

Only one retrospective non-randomized study concerning renal transplant was included in our analysis, which was conducted in Turkey and compared robotic with open renal transplant. ${ }^{88}$ It included 80 adult patients (53 men, 27 women). All three anastomoses (arterial, venous, ureterovesical) were performed faster with the open than the robotic approach. In particular, the mean (SD) anastomotic times for arterial, venous and ureterovesical anastomoses

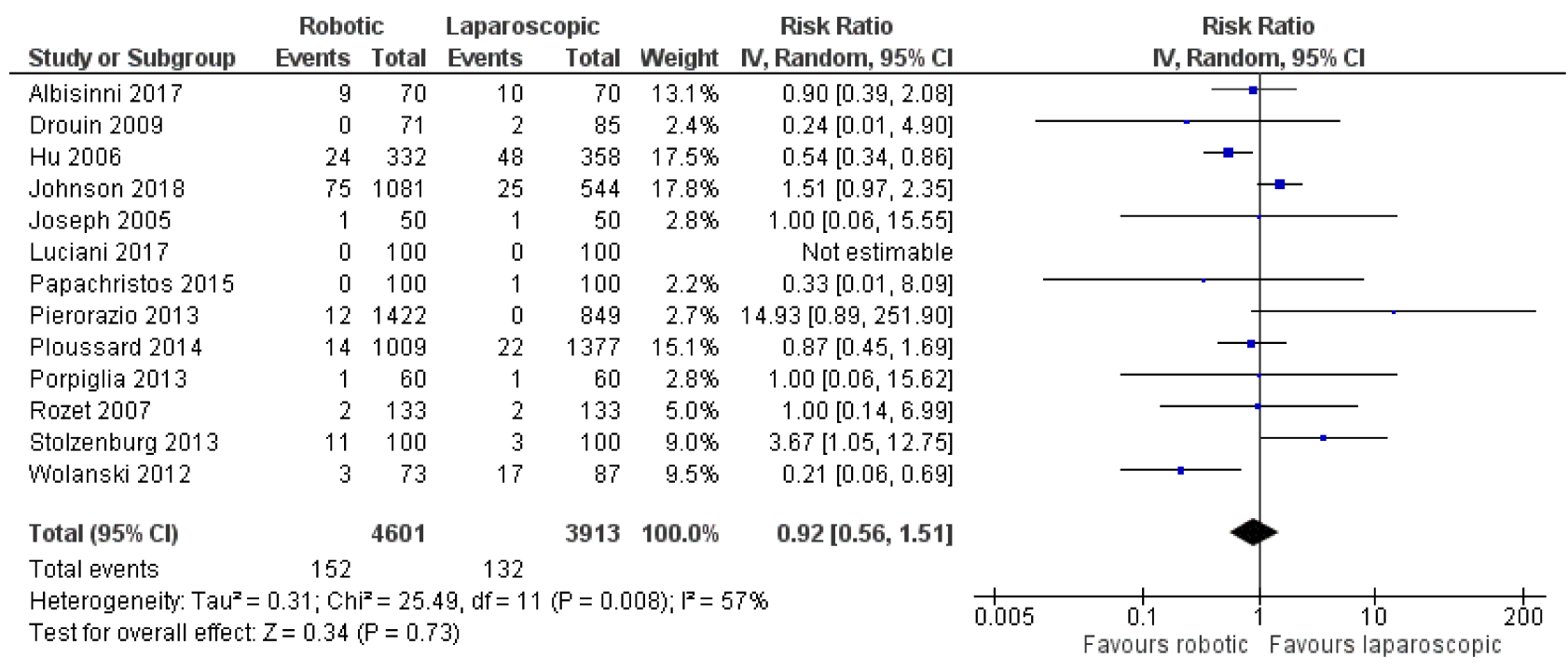

Figure 14 Comparison between robotic and laparoscopic prostatectomy: anastomotic leak. 


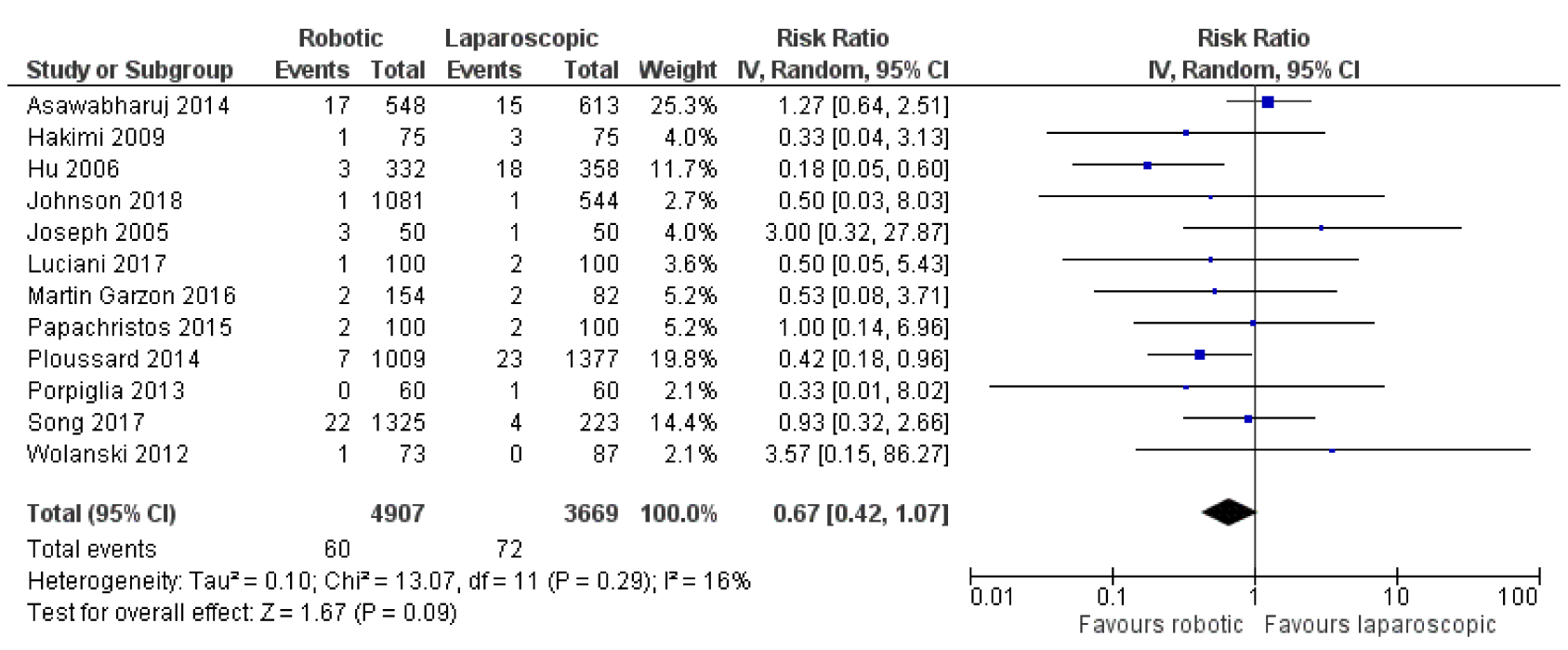

Figure 15 Comparison between robotic and laparoscopic prostatectomy: anastomotic stricture/contracture.

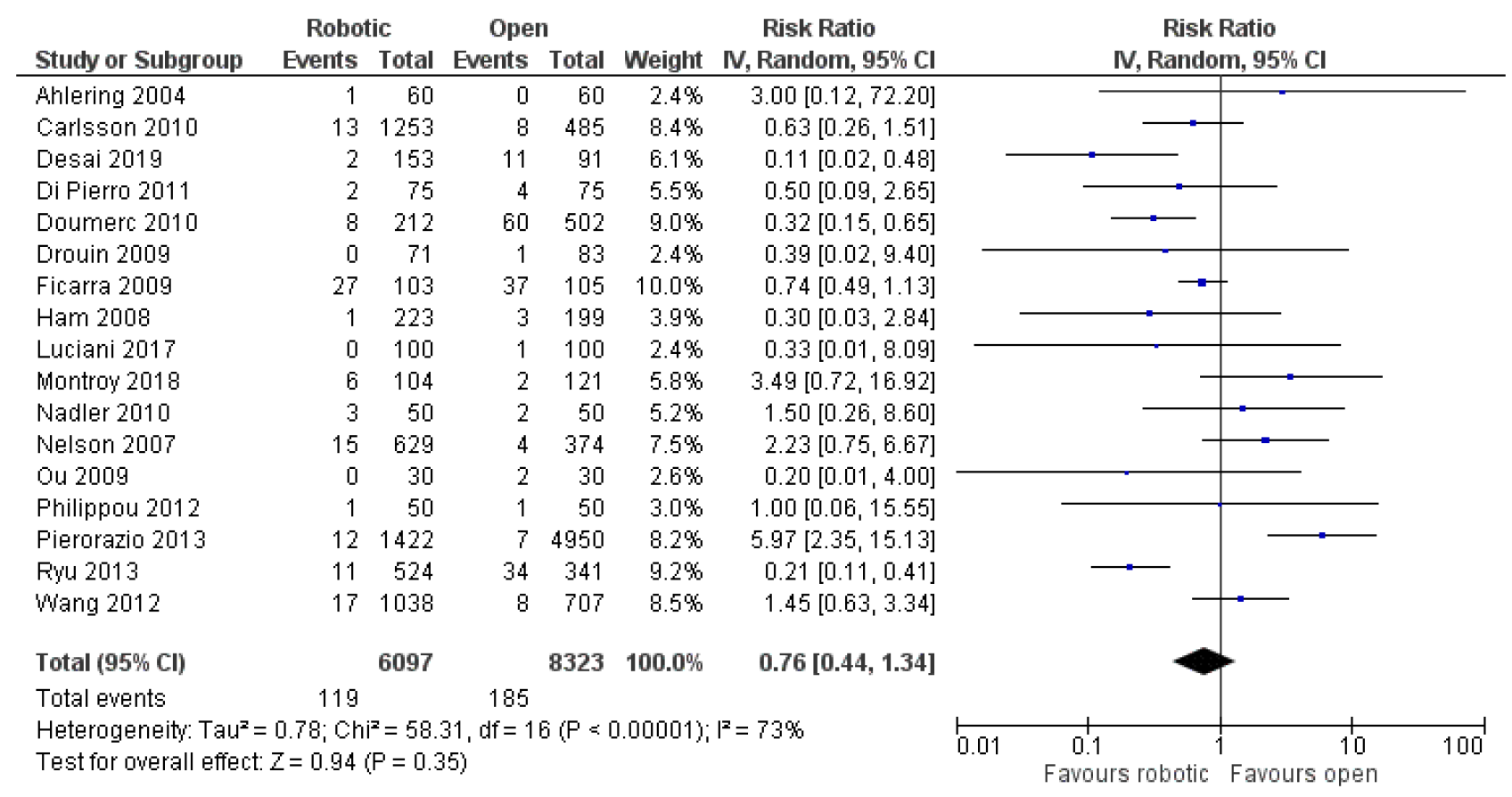

Figure 16 Comparison between robotic and open radical prostatectomy: anastomotic leak.

were $18.45 \min (5.73), 20.92 \min (6.57)$ and $21.30 \mathrm{~min}$ (4.73), respectively, in robotic cases, whereas they were $14.97 \mathrm{~min}$ (2.59), $16.02 \mathrm{~min}$ (2.3) and $14.95 \mathrm{~min}$ (1.56), respectively, in open cases. All differences were statistically significant $(\mathrm{p} \leq 0.001)$. There was no available information about anastomotic leaks/bleeding or strictures. ${ }^{88}$

\section{Discussion}

Since its introduction almost 20 years ago, robotic surgery has been successfully applied to key colorectal, gastric, pancreatic, urological and transplantation procedures where anastomoses form a critical part of the operation. Anastomoses are often time critical, in particular in transplantation where minimising warm ischaemia of the organ is critical to the graft function and outcome. ${ }^{89,90}$ Furthermore, anastomotic complications such as leak of urine or intestinal contents result in significant morbidity including the need for salvage surgery. The incidence of strictures or stenoses is also of importance in the functional outcome of an anastomosis and may necessitate 


\begin{tabular}{|c|c|c|c|c|c|c|c|c|c|c|}
\hline \multirow[b]{2}{*}{ Study or Subgroup } & \multicolumn{2}{|c|}{ Robotic } & \multicolumn{2}{|c|}{ Open } & \multicolumn{3}{|c|}{ Risk Ratio } & \multirow{2}{*}{\multicolumn{2}{|c|}{$\begin{array}{c}\text { Risk Ratio } \\
\text { IV, Random, } 95 \% \mathrm{Cl}\end{array}$}} & \\
\hline & Events & Total & Events & Total & Weight & IV, Random, 95\% Cl & & & & \\
\hline Breyer 2010 & 4 & 293 & 18 & 695 & $6.6 \%$ & $0.53[0.18,1.54]$ & & & & \\
\hline Carlsson 2010 & 3 & 1253 & 22 & 485 & $5.6 \%$ & $0.05[0.02,0.18]$ & & & & \\
\hline Di Pierro 2011 & 1 & 75 & 7 & 75 & $2.3 \%$ & $0.14[0.02,1.13]$ & & & & \\
\hline Ficarra 2009 & 3 & 103 & 6 & 105 & $4.7 \%$ & $0.51[0.13,1.98]$ & & & & \\
\hline Gontero 2019 & 16 & 209 & 31 & 186 & $13.1 \%$ & $0.46[0.26,0.81]$ & & $\longrightarrow-$ & & \\
\hline $\mathrm{Hu} 200 \mathrm{~g}$ & 99 & 1938 & 946 & 6899 & $19.8 \%$ & $0.37[0.30,0.46]$ & & - & & \\
\hline Krambeck 2009 & 13 & 294 & 33 & 588 & $12.1 \%$ & $0.79[0.42,1.47]$ & & & & \\
\hline Luciani 2017 & 1 & 100 & 1 & 100 & $1.4 \%$ & $1.00[0.06,15.77]$ & & & & \\
\hline Wontroy 2018 & 0 & 104 & 7 & 121 & $1.3 \%$ & $0.08[0.00,1.34]$ & & & & \\
\hline Nadler 2010 & 3 & 50 & 7 & 50 & $5.0 \%$ & $0.43[0.12,1.56]$ & & & - & \\
\hline Ou 2009 & 1 & 30 & 0 & 30 & $1.1 \%$ & $3.00[0.13,70.83]$ & & & & \\
\hline Philippou 2012 & 2 & 50 & 4 & 50 & $3.4 \%$ & $0.50[0.10,2.61]$ & & $\rightarrow$ & & \\
\hline Song 2017 & 22 & 1325 & 35 & 1069 & $13.9 \%$ & $0.51[0.30,0.86]$ & & $\rightarrow-$ & & \\
\hline Wallerstedt Lant 2019 & 2 & 2764 & 2 & 942 & $2.5 \%$ & $0.34[0.05,2.42]$ & & & & \\
\hline Wang 2012 & 10 & 1038 & 6 & 707 & $7.2 \%$ & $1.14[0.41,3.11]$ & & & & \\
\hline Total $(95 \% \mathrm{Cl})$ & & 9626 & & 12102 & $100.0 \%$ & $0.44[0.32,0.62]$ & & & & \\
\hline Total events & 180 & & 1125 & & & & & & & \\
\hline $\begin{array}{l}\text { Heterogeneity: Tau }{ }^{2}=0 \\
\text { Test for overall effect: } Z\end{array}$ & $\begin{array}{l}3 ; \mathrm{Ch}^{2}= \\
4.78(\mathrm{P}=\end{array}$ & $\begin{array}{l}25.56 \\
0.0000\end{array}$ & $\mathrm{df}=14(\mathrm{f}$ & $=0.03)$ & $i^{2}=45 \%$ & & 0.005 & $\begin{array}{c}0.1 \\
\text { Favours robotic }\end{array}$ & $1 \begin{array}{r}10 \\
\text { Favours of }\end{array}$ & 200 \\
\hline
\end{tabular}

Figure 17 Comparison between robotic and open radical prostatectomy: anastomotic stricture/contracture.

intervention or even revision surgery. ${ }^{91}$ The integrity of the anastomosis, therefore, may represent a crucial factor when comparing the overall benefits of a robotic surgical approach to laparoscopic or open alternatives.

In most comparisons, the robotic approach appears to provide similar results with the laparoscopic and open approaches in terms of anastomotic leak or stenosis. In particular, when we compared robotically performed anastomoses with laparoscopically performed anastomoses, no significant differences were found concerning leak or stricture after gastrectomy, leak after RYGB, pancreatic leak or bile leak after pancreaticoduodenectomy, leak or stricture/failure after pyeloplasty, and leak or stricture/contracture after radical prostatectomy. On the other hand, there were lower rates of anastomotic stricture after RYGB with the robotic technique. As far as the comparison of anastomoses performed with the robotic approach with those performed with the open approach, no significant differences were detected regarding leak or stricture after RYGB, pancreatic leak, bile leak or gastrointestinal leak after pancreaticoduodenectomy, leak or stricture/failure after pyeloplasty, leak after radical cystectomy, and leak after radical prostatectomy. On the contrary, there were lower rates of anastomotic stricture/contracture after radical prostatectomy with the robotic technique. Furthermore, it appears that all anastomoses (arterial, venous, ureterovesical) in renal transplant are performed more quickly via the open than the robotic approach. To conclude, robotically performed anastomoses provide similar results to those performed via laparoscopic or open approach, with the exception of RYGB, where the incidence of stenosis is less frequent in robotic than in laparoscopic operations, radical prostatectomy, where stenosis is less common in robotic than in open operations, and renal transplant, where the duration of anastomoses is longer in the robotic than the open approach.

It is important to note that the main focus of this analysis was a comparison of the anastomotic technique employed in these procedures, and the relevant anastomosis-related complications, which are deemed to confer significant morbidity, particularly in the early and mid-term post-operative period. This represents one of the limitations of this review, as it does not take into account oncological outcomes, incidence of nerve damage, incision size or incidence of incision-related complications, or ease of access, in particular for deep pelvic surgery. These are all factors that would undoubtedly contribute heavily when determining the overall benefit and/or superiority of robotic approaches as compared to open or laparoscopic approaches. For example, in robotic radical prostatectomy, superior vision and more intricate operating, facilitated by the robotic platform, has enabled improved nerve-sparing techniques resulting in superior functional outcomes, with no compromise in oncological outcomes. ${ }^{92}$ Our review also excluded procedures where a significant part of the operation is performed via a robotic approach, but not the relevant anastomosis - for example, in low rectal surgery for 
cancer. Current evidence suggests that the robotic approach for rectal cancer is of particular benefit in difficult cases such as patients with previous abdominal surgery and chemoradiation therapy. ${ }^{93}$ Lower conversion rates to open surgery have also been reported, ${ }^{94}$ as well as comparable, ${ }^{95}$ if not superior oncological outcomes than laparoscopic approaches, and significantly better autonomic functional outcomes. $^{96}$ Furthermore, when comparing the overall advantages of robotic surgery to open or laparoscopic alternatives, cost-effectiveness is undoubtedly a key consideration. However, in many cases, the increased costs associated with a robotic system and its maintenance may well be offset by a shorter length of hospital stay and lower complication rates. $^{97-100}$

In conclusion, this review shows equivalent outcomes of robotically performed anastomoses with those performed via laparoscopic or open approach, apart from the few aforementioned exceptions. This means that the other benefits of robotic operations are not compromised by any deficit in anastomotic outcomes. This is with particular reference to abdominal operations where the anastomoses and its related complications are deemed to be a significant factor contributing to the overall morbidity and outcome. The choice of robotic over current gold-standard approaches for these operations, therefore, may lie with other demonstrable benefits such as superior vision, better access to the pelvis, and superior functional or oncological outcomes, as briefly discussed above.

\section{Disclosure}

Ioannis Loukopoulos reports grants from Intuitive Surgical Operations Inc., during the conduct of the study. Nicos Kessaris reports grants from Intuitive Surgical (Clinical Research Grant), outside the submitted work. The authors report no other conflicts of interest in this work.

\section{References}

1. Ruurda JP, Can Vroonhoven TJ, Broeders IA. Robot-assisted surgical systems: a new era in laparoscopic surgery. Ann R Coll Surg Engl. 2002;84:223-226. doi:10.1308/003588402320439621

2. Lanfranco AR, Castellanos AE, Desai JP, et al. Robotic surgery: a current perspective. Ann Surg. 2004;239:14-21. doi:10.1097/01. sla.0000103020.19595.7d

3. Prasad SM, Ducko CT, Stephenson ER, et al. Prospective clinical trial of robotically assisted endoscopic coronary grafting with 1 year follow-up. Ann Surg. 2001;233:725-732. doi:10.1097/00000658200106000-00001

4. Pein U, Girndt M, Markau S, et al. Minimally invasive robotic versus conventional open living donor kidney transplantation. World J Urol. 2019. doi:10.1007/s00345-019-02814-7

5. Moher D, Liberati A, Tetzlaff J, et al. Preferred reporting items for systematic reviews and meta-analyses: the PRISMA statement. PLoS Med. 2009;6:e1000097. doi:10.1371/journal.pmed.1000097
6. Caruso R, Vicente E, Quijano Y, et al. Robotic assisted gastrectomy compared with open resection: a case-matched study. Updates Surg. 2019;71:367-373. doi:10.1007/s13304-018-0533-5

7. Pan HF, Wang G, Liu J, et al. Robotic versus laparoscopic gastrectomy for locally advanced gastric cancer. Surg Laparosc Endosc Percutan Tech. 2017;27:428-433. doi:10.1097/ SLE.0000000000000469

8. Solaini L, Bazzocchi F, Pellegrini S, et al. Robotic vs open gastrectomy for gastric cancer: a propensity score-matched analysis on short- and long-term outcomes. Int J Med Robot. 2019;15 (5):e2019. [Epub ahead of print].

9. Wang G, Jiang Z, Zhao J, et al. Assessing the safety and efficacy of full robotic gastrectomy with intracorporeal robot-sewn anastomosis for gastric cancer: a randomized clinical trial. J Surg Oncol. 2016;113:397-404. doi:10.1002/jso.v113.4

10. Ahmad A, Carleton JD, Ahmad ZF, et al. Laparoscopic versus robotic-assisted Roux-en- $Y$ gastric bypass: a retrospective, single-center study of early perioperative outcomes at a community hospital. Surg Endosc. 2016;30:3792-3796. doi:10.1007/s00464-015-4675-y

11. Ayloo S, Roh Y, Choudhury N. Laparoscopic, hybrid, and totally robotic Roux-en-Y gastric bypass. J Robot Surg. 2016;10:41-47. doi:10.1007/s11701-016-0559-y

12. Hagen ME, Pugin F, Chassot G, et al. Reducing cost of surgery by avoiding complications: the model of robotic Roux-en-Y gastric bypass. Obes Surg. 2012;22:52-61. doi:10.1007/s11695-0110422-1

13. Rogula T, Koprivanac M, Janik MR, et al. Does robotic Roux-enY gastric bypass provide outcome advantages over standard laparoscopic approaches? Obes Surg. 2018;28:2589-2596. doi:10.1007/s11695-018-3228-6

14. Sanchez BR, Mohr CJ, Morton JM, et al. Comparison of totally robotic laparoscopic Roux-en-Y gastric bypass and traditional laparoscopic Roux-en-Y gastric bypass. Surg Obes Relat Dis. 2005;1:549-554. doi:10.1016/j.soard.2005.08.008

15. Baker EH, Ross SW, Seshadri R, et al. Robotic pancreaticoduodenectomy: comparison of complications and cost to the open approach. Int J Med Robot. 2016;12:554-560. doi:10.1002/rcs. v12.3

16. Bao PQ, Mazirka PO, Watkins KT. Retrospective comparison of robot-assisted minimally invasive versus open pancreaticoduodenectomy for periampullary neoplasms. $J$ Gastrointest Surg. 2014;18:682-689. doi:10.1007/s11605-013-2410-3

17. Boggi U, Napoli N, Costa F, et al. Robotic-assisted pancreatic resections. World J Surg. 2016;40:2497-2506. doi:10.1007/ s00268-016-3565-3

18. Buchs NC, Addeo P, Bianco FM, et al. Robotic versus open pancreaticoduodenectomy: a comparative study at a single institution. World J Surg. 2011;35:2739-2746. doi:10.1007/ s00268-011-1276-3

19. Chalikonda S, Aguilar-Saavedra JR, Walsh RM. Laparoscopic robotic-assisted pancreaticoduodenectomy: a case-matched comparison with open resection. Surg Endosc. 2012;26:2397-2402. doi:10.1007/s00464-012-2207-6

20. Chen S, Chen JZ, Zhan Q, et al. Robot-assisted laparoscopic versus open pancreaticoduodenectomy: a prospective, matched, mid-term follow-up study. Surg Endosc. 2015;29:3698-3711. doi:10.1007/s00464-015-4140-y

21. Kim HS, Han Y, Kang JS, et al. Comparison of surgical outcomes between open and robot-assisted minimally invasive pancreaticoduodenectomy. $J$ Hepatobiliary Pancreat Sci. 2018;25:142-149. doi:10.1002/jhbp.522

22. Lai EC, Yang GP, Tang CN. Robot-assisted laparoscopic pancreaticoduodenectomy versus open pancreaticoduodenectomy a comparative study. Int J Surg. 2012;10:475-479. doi:10.1016/ j.ijsu.2012.06.003 
23. Liu R, Zhang T, Zhao ZM, et al. The surgical outcomes of robot-assisted laparoscopic pancreaticoduodenectomy versus laparoscopic pancreaticoduodenectomy for periampullary neoplasms: a comparative study of a single center. Surg Endosc. 2017;31:2380-2386. doi:10.1007/s00464-016-5238-6

24. McMillan MT, Zureikat AH, Hogg ME, et al. A propensity score-matched analysis of robotic vs open pancreatoduodenectomy on incidence of pancreatic fistula. JAMA Surg. 2017;152:327-335. doi:10.1001/jamasurg.2016.4755

25. Nassour I, Wang SC, Porembka MR, et al. Robotic versus laparoscopic pancreaticoduodenectomy: a NSQIP analysis. $J$ Gastrointest Surg. 2017;21:1784-1792. doi:10.1007/s11605017-3543-6

26. Varley PR, Zenati MS, Klobuka A, et al. Does robotic pancreaticoduodenectomy improve outcomes in patients with high risk morphometric features compared to the open approach? $H P B$ (Oxford). 2019;21:695-701. doi:10.1016/j.hpb.2018.10.016

27. Zhang Y, Hong D, Zhang C, et al. Total laparoscopic versus robot-assisted laparoscopic pancreaticoduodenectomy. Biosci Trends. 2018;12:484-490. doi:10.5582/bst.2018.01236

28. Zhou NX, Chen JZ, Liu Q, et al. Outcomes of pancreatoduodenectomy with robotic surgery versus open surgery. Int $J$ Med Robot. 2011;7:131-137. doi:10.1002/rcs.380

29. Zimmerman AM, Roye DG, Charpentier KP. A comparison of outcomes between open, laparoscopic and robotic pancreaticoduodenectomy. HPB (Oxford). 2018;20:364-369. doi:10.1016/j.hpb.2017.10.008

30. Zureikat AH, Postlewait LM, Liu Y, et al. A multi-institutional comparison of perioperative outcomes of robotic and open pancreaticoduodenectomy. Ann Surg. 2016;264:640-649. doi:10.1097/SLA.0000000000001869

31. Sung HH, Ahn JS, Seo SI, et al. A comparison of early complications between open and robot-assisted radical cystectomy. J Endourol. 2012;26:670-675. doi:10.1089/end.2011.0372

32. Bansal D, Cost NG, DeFoor WR Jr, et al. Infant robotic pyeloplasty: comparison with an open cohort. $J$ Pediatr Urol. 2014;10:380-385. doi:10.1016/j.jpurol.2013.10.016

33. Barbosa JA, Kowal A, Onal B, et al. Comparative evaluation of the resolution of hydronephrosis in children who underwent open and robotic-assisted laparoscopic pyeloplasty. J Pediatr Urol. 2013;9:199-205. doi:10.1016/j.jpurol.2012.02.002

34. Başataç C, Boylu U, Önol FF, et al. Comparison of surgical and functional outcomes of open, laparoscopic and robotic pyeloplasty for the treatment of ureteropelvic junction obstruction. Turk J Urol. 2014;40:24-30. doi:10.5152/tud.2014.06956

35. Bernie JE, Venkatesh R, Brown J, et al. Comparison of laparoscopic pyeloplasty with and without robotic assistance. JSLS. 2005;9:258-261.

36. Faddegon S, Granberg C, Tan YK, et al. Minimally invasive pyeloplasty in horseshoe kidneys with ureteropelvic junction obstruction: a case series. Int Braz J Urol. 2013;39:195-202. doi:10.1590/S1677-5538.IBJU.2013.02.07

37. Fiori C, Bertolo R, Manfredi M, et al. Robot-assisted laparoendoscopic single-site versus mini-laparoscopic pyeloplasty: a comparison of perioperative, functional and cosmetic results. Minerva Urol Nefrol. 2017;69:604-612. doi:10.23736/S03932249.17.02833-8

38. Franco I, Dyer LL, Zelkovic P. Laparoscopic pyeloplasty in the pediatric patient: hand sewn anastomosis versus robotic assisted anastomosis - is there a difference? J Urol. 2007;178:1483-1486. doi:10.1016/j.juro.2007.06.012

39. Hemal AK, Mukherjee S, Singh K. Laparoscopic pyeloplasty versus robotic pyeloplasty for ureteropelvic junction obstruction: a series of 60 cases performed by a single surgeon. Can J Urol. 2010;17:5012-5016.
40. Hong P, Ding G, Zhu D, et al. Head-to-head comparison of modified laparoscopic pyeloplasty and robot-assisted pyeloplasty for ureteropelvic junction obstruction in China. Urol Int. 2018;101:337-344. doi:10.1159/000492337

41. Kumar R, Nayak B. Robotic versus conventional laparoscopic pyeloplasty: a single surgeon concurrent cohort review. Indian J Urol. 2013;29:19-21. doi:10.4103/0970-1591.109978

42. Link RE, Bhayani SB, Kavoussi LR. A prospective comparison of robotic and laparoscopic pyeloplasty. Ann Surg. 2006;243:486-491. doi:10.1097/01.sla.0000205626.71982.32

43. Neheman A, Kord E, Zisman A, et al. Comparison of robotic pyeloplasty and standard laparoscopic pyeloplasty in infants: a bi-institutional study. $J$ Laparoendosc Adv Surg Tech A. 2018;28:467-470. doi:10.1089/lap.2017.0262

44. Olweny EO, Park SK, Tan YK, et al. Perioperative comparison of robotic assisted laparoendoscopic single-site (LESS) pyeloplasty versus conventional LESS pyeloplasty. Eur Urol. 2012;61:410-414. doi:10.1016/j.eururo.2011.10.024

45. Riachy E, Cost NG, Defoor WR, et al. Pediatric standard and robot-assisted laparoscopic pyeloplasty: a comparative single institution study. $J$ Urol. 2013;189:283-287. doi:10.1016/j. juro.2012.09.008

46. Song SH, Lee C, Jung J, et al. A comparative study of pediatric open pyeloplasty, laparoscopy-assisted extracorporeal pyeloplasty, and robot-assisted laparoscopic pyeloplasty. PLoS One. 2017;12:e0175026. doi:10.1371/journal.pone.0175026

47. Sorensen MD, Delostrinos C, Johnson MH, et al. Comparison of the learning curve and outcomes of robotic assisted pediatric pyeloplasty. J Urol. 2011;185:2517-2522. doi:10.1016/j.juro.2011.01.021

48. Subotic U, Rohard I, Weber DM, et al. A minimal invasive surgical approach for children of all ages with ureteropelvic junction obstruction. $J$ Pediatr Urol. 2012;8:354-358. doi:10.1016/j.jpurol.2011.07.004

49. Tam YH, Pang KKY, Wong YS, et al. From laparoscopic pyeloplasty to robot-assisted laparoscopic pyeloplasty in primary and reoperative repairs for ureteropelvic junction obstruction in children. J Laparoendosc Adv Surg Tech A. 2018;28:1012-1018. doi:10.1089/lap.2017.0561

50. Weise ES, Winfield HN. Robotic computer-assisted pyeloplasty versus conventional laparoscopic pyeloplasty. $J$ Endourol. 2006;20:813-819. doi:10.1089/end.2006.20.813

51. Yee DS, Shanberg AM, Duel BP, et al. Initial comparison of robotic-assisted laparoscopic versus open pyeloplasty in children. Urology. 2006;67:599-602. doi:10.1016/j.urology.2005. 09.021

52. Ahlering TE, Woo D, Eichel L, et al. Robot-assisted versus open radical prostatectomy: a comparison of one surgeon's outcomes. Urology. 2004;63:819-822. doi:10.1016/j.urology.2004.01.038

53. Albisinni S, Aoun F, LE DD, et al. Comparing conventional laparoscopic to robotic-assisted extended pelvic lymph node dissection in men with intermediate and high-risk prostate cancer: a matched-pair analysis. Minerva Urol Nefrol. 2017;69:101-107. doi:10.23736/S0393-2249.16.02799-5

54. Asawabharuj K, Ramart P, Nualyong C, et al. Comparison of urinary continence outcome between robotic assisted laparoscopic prostatectomy versus laparoscopic radical prostatectomy. $J$ Med Assoc Thai. 2014;97:393-398.

55. Breyer BN, Davis CB, Cowan JE, et al. Incidence of bladder neck contracture after robot-assisted laparoscopic and open radical prostatectomy. BJU Int. 2010;106:1734-1738. doi:10.1111/ bju.2010.106.issue-11

56. Carlsson S, Nilsson AE, Schumacher MC, et al. Surgery-related complications in 1253 robot-assisted and 485 open retropubic radical prostatectomies at the Karolinska University Hospital, Sweden. Urology. 2010;75:1092-1097. doi:10.1016/j.urology.2009.09.075 
57. Desai A, Hudnall M, Weiner AB, et al. Contemporary comparison of open to robotic prostatectomy at a veteran's affairs hospital. Mil Med. 2019;184:e330-e337. doi:10.1093/milmed/usy352

58. Di Pierro GB, Baumeister P, Stucki P, et al. A prospective trial comparing consecutive series of open retropubic and robot-assisted laparoscopic radical prostatectomy in a centre with a limited caseload. Eur Urol. 2011;59:1-6. doi:10.1016/j. eururo.2010.10.026

59. Doumerc N, Yuen C, Savdie R, et al. Should experienced open prostatic surgeons convert to robotic surgery? The real learning curve for one surgeon over 3 years. BJU Int. 2010;106:378-384. doi:10.1111/j.1464-410X.2009.09158.x

60. Drouin SJ, Vaessen C, Hupertan V, et al. Comparison of mid-term carcinologic control obtained after open, laparoscopic, and robot-assisted radical prostatectomy for localized prostate cancer. World J Urol. 2009;27:599-605. doi:10.1007/s00345009-0379-z

61. Ficarra V, Novara G, Fracalanza S, et al. A prospective, non-randomized trial comparing robot-assisted laparoscopic and retropubic radical prostatectomy in one European institution. BJU Int. 2009;104:534-539. doi:10.1111/j.1464-410X.2009.08419.X

62. Gontero P, Marra G, Alessio P, et al. Salvage radical prostatectomy for recurrent prostate cancer: morbidity and functional outcomes from a large multicenter series of open versus robotic approaches. J Urol. 2019;202:725-731. doi:10.1097/JU.0000 000000000327

63. Hakimi AA, Blitstein J, Feder M, et al. Direct comparison of surgical and functional outcomes of robotic-assisted versus pure laparoscopic radical prostatectomy: single-surgeon experience. Urology. 2009;73:119-123. doi:10.1016/j.urology.2008.08.491

64. Ham WS, Park SY, Kim WT, et al. Open versus robotic radical prostatectomy: a prospective analysis based on a single surgeon's experience. J Robot Surg. 2008;2:235-241. doi:10.1007/s11701008-0111-9

65. Hu JC, Nelson RA, Wilson TG, et al. Perioperative complications of laparoscopic and robotic assisted laparoscopic radical prostatectomy. J Urol. 2006;175:541-546. doi:10.1016/S00225347(05)00156-4

66. $\mathrm{Hu} \mathrm{JC}, \mathrm{Gu} \mathrm{X}$, Lipsitz SR, et al. Comparative effectiveness of minimally invasive vs open radical prostatectomy. JAMA. 2009;302:1557-1564. doi:10.1001/jama.2009.1451

67. Johnson I, Ottosson F, Diep LM, et al. Switching from laparoscopic radical prostatectomy to robot assisted laparoscopic prostatectomy: comparing oncological outcomes and complications. Scand J Urol. 2018;52:116-121. doi:10.1080/21681805.2017. 1420099

68. Joseph JV, Vicente I, Madeb R, et al. Robot-assisted vs pure laparoscopic radical prostatectomy: are there any differences? BJU Int. 2005;96:39-42. doi:10.1111/bju.2005.96.issue-1

69. Krambeck AE, DiMarco DS, Rangel LJ, et al. Radical prostatectomy for prostatic adenocarcinoma: a matched comparison of open retropubic and robot-assisted techniques. BJU Int. 2009;103:448-453. doi:10.1111/j.1464-410X.2008.08012.x

70. Luciani LG, Mattevi D, Mantovani W, et al. Retropubic, laparoscopic, and robot-assisted radical prostatectomy: a comparative analysis of the surgical outcomes in a single regional center. Curr Urol. 2017;11:36-41. doi:10.1159/000447192

71. Martín Garzón OD, Azhar RA, Brunacci L, et al. One-year outcome comparison of laparoscopic, robotic, and robotic intrafascial simple prostatectomy for benign prostatic hyperplasia. J Endourol. 2016;30:312-318. doi:10.1089/end.2015.0218

72. Montroy J, Elzayat E, Morash C, et al. Long-term patient outcomes from the first year of a robotic surgery program using multi-surgeon implementation. Can Urol Assoc J. 2018;12:38-43. doi:10.5489/ cuaj. 4528
73. Nadler RB, Casey JT, Zhao LC, et al. Is the transition from open to robotic prostatectomy fair to your patients? A single-surgeon comparison with 2-year follow-up. $J$ Robot Surg. 2010;3:201-207. doi:10.1007/s11701-009-0162-6

74. Nelson B, Kaufman M, Broughton G, et al. Comparison of length of hospital stay between radical retropubic prostatectomy and robotic assisted laparoscopic prostatectomy. $J$ Urol. 2007;177:929-931. doi:10.1016/j.juro.2006.10.070

75. Ou YC, Yang CR, Wang J, et al. Comparison of robotic-assisted versus retropubic radical prostatectomy performed by a single surgeon. Anticancer Res. 2009;29:1637-1642.

76. Papachristos A, Basto M, Te Marvelde L, et al. Laparoscopic versus robotic-assisted radical prostatectomy: an Australian single-surgeon series. ANZ J Surg. 2015;85:154-158. doi:10.1111/ans.2015.85.issue-3

77. Philippou P, Waine E, Rowe E. Robot-assisted laparoscopic prostatectomy versus open: comparison of the learning curve of a single surgeon. J Endourol. 2012;26:1002-1008. doi:10.1089/ end.2011.0569

78. Pierorazio PM, Mullins JK, Ross AE, et al. Trends in immediate perioperative morbidity and delay in discharge after open and minimally invasive radical prostatectomy (RP): a 20-year institutional experience. BJU Int. 2013;112:45-53. doi:10.1111/j.1464410X.2012.11767.x

79. Ploussard G, de la Taille A, Moulin M, et al. Comparisons of the perioperative, functional, and oncologic outcomes after robot-assisted versus pure extraperitoneal laparoscopic radical prostatectomy. Eur Urol. 2014;65:610-619. doi:10.1016/j. eururo.2012.11.049

80. Porpiglia F, Morra I, Lucci Chiarissi M, et al. Randomised controlled trial comparing laparoscopic and robot-assisted radical prostatectomy. Eur Urol. 2013;63:606-614. doi:10.1016/j. eururo.2012.07.007

81. Rozet F, Jaffe J, Braud G, et al. A direct comparison of robotic assisted versus pure laparoscopic radical prostatectomy: a single institution experience. J Urol. 2007;178:478-482. doi:10.1016/j. juro.2007.03.111

82. Ryu J, Kwon T, Kyung YS, et al. Retropubic versus robot-assisted laparoscopic prostatectomy for prostate cancer: a comparative study of postoperative complications. Korean $J$ Urol. 2013;54:756-761. doi:10.4111/kju.2013.54.11.756

83. Song W, Park JH, Jeon HG, et al. Comparison of oncologic outcomes and complications according to surgical approach to radical prostatectomy: special focus on the perineal approach. Clin Genitourin Cancer. 2017;15:e645-e652. doi:10.1016/j. clgc.2017.01.015

84. Stolzenburg JU, Qazi HA, Holze S, et al. Evaluating the learning curve of experienced laparoscopic surgeons in robot-assisted radical prostatectomy. J Endourol. 2013;27:80-85. doi:10.1089/ end.2012.0262

85. Wallerstedt Lantz A, Stranne J, Tyritzis SI, et al. 90-day readmission after radical prostatectomy-a prospective comparison between robot-assisted and open surgery. Scand J Urol. 2019;53:26-33. doi:10.1080/21681805.2018.1556729

86. Wang R, Wood DP Jr, Hollenbeck BK, et al. Risk factors and quality of life for post-prostatectomy vesicourethral anastomotic stenoses. Urology. 2012;79:449-457. doi:10.1016/j.urology.2011.07.1383

87. Wolanski P, Chabert C, Jones L, et al. Preliminary results of robot-assisted laparoscopic radical prostatectomy (RALP) after fellowship training and experience in laparoscopic radical prostatectomy (LRP). BJU Int. 2012;110:64-70. doi:10.1111/ bju.2012.110.issue-s4

88. Tuğcu V, Şener NC, Şahin S, et al. Robot-assisted kidney transplantation: comparison of the first 40 cases of open vs robot-assisted transplantations by a single surgeon. BJU Int. 2018;121:275-280. doi:10.1111/bju.2018.121.issue-2 
89. Ferede AA, Walsh AL, Davis NF, et al. Warm ischemia time at vascular anastomosis is an independent predictor for delayed graft function in kidney transplant recipients. Exp Clin Transplant. 2019. doi:10.6002/ect

90. Heylen L, Pirenne J, Samuel U, et al. The impact of anastomosis time during kidney transplantation on graft loss: a eurotransplant cohort study. Am J Transplant. 2017;17:724-732. doi:10.1111/ajt.2017.17. issue-3

91. Guyton KL, Hyman NH, Alverdy JC. Prevention of perioperative anastomotic healing complications: anastomotic stricture and anastomotic leak. Adv Surg. 2016;50:129-141. doi:10.1016/j. yasu.2016.03.011

92. McGuiness LA, Rai BP. Robotics in urology. Ann $R$ Coll Surg Engl. 2018;100:45-54. doi:10.1308/rcsann.supp1.38

93. Scarpinata R, Aly EH. Does robotic rectal cancer surgery offer improved early postoperative outcomes? Dis Colon Rectum. 2013;56:253-262. doi:10.1097/DCR.0b013e3182694595

94. Ackerman SJ, Daniel S, Baik R, et al. Comparison of complication and conversion rates between robotic-assisted and laparoscopic rectal resection for rectal cancer: which patients and providers could benefit most from robotic-assisted surgery? J Med Econ. 2018;21:254-261. doi:10.1080/13696998.2017.1396994
95. Baek JH, McKenzie S, Garcia-Aguilar J, et al. Oncologic outcomes of robotic-assisted total mesorectal excision for the treatment of rectal cancer. Ann Surg. 2010;251:882-886. doi:10.1097/ SLA.0b013e3181c79114

96. D’Annibale A, Pernazza G, Monsellato I, et al. Total mesorectal excision: a comparison of oncological and functional outcomes between robotic and laparoscopic surgery for rectal cancer. Surg Endosc. 2013;27:1887-1895. doi:10.1007/s00464012-2731-4

97. Tyler JA, Fox JP, Desai MM, et al. Outcomes and costs associated with robotic colectomy in the minimally invasive era. Dis Colon Rectum. 2013;56:458-466. doi:10.1097/DCR.0b013e31827085ec

98. Halabi WJ, KAng CY, Jafari MD, et al. Robotic assisted colorectal surgery in the United States: a nationwide analysis of trends and outcomes. World J Surg. 2012;37:2782-2790. doi:10.1007/ s00268-013-2024-7

99. Sivathondan PC, Jayne DG. The role of robotics in colorectal surgery. Ann R Coll Surg Engl. 2018;100:42-53. doi:10.1308/ rcsann.supp 2.42

100. Khosla A, Wagner AA. Robotic surgery of the kidney, bladder and prostate. Surg Clin N Am. 2016;96:615-636. doi:10.1016/j. suc.2016.02.015

\section{Publish your work in this journal}

Robotic Surgery: Research and Reviews is an international, peer reviewed, open access, online journal publishing original research, commentaries, reports, and reviews on the theory, use and application of robotics in surgical interventions. Articles on the use of supervisorycontrolled robotic systems, telesurgical devices, and shared-control systems are invited. The manuscript management system is completely online and includes a very quick and fair peer review system, which is all easy to use. Visit http://www.dovepress.com/testimonials. php to read real quotes from published authors. 